\title{
ON INEQUALITIES FOR SUMS OF BOUNDED RANDOM VARIABLES
}

\author{
IOSIF PINELIS
}

\begin{abstract}
A new upper bound on $\mathrm{P}\left(a_{1} \eta_{1}+a_{2} \eta_{2}+\ldots \geqslant x\right)$ is obtained, where $\eta_{1}, \eta_{2}, \ldots$ are independent zero-mean random variables such that $\left|\eta_{i}\right| \leqslant 1$ for all $i$. A multidimensional analogue of this result and extensions to (super)martingales are presented, as well as an application to self-normalized sums (or, equivalently, to $t$-statistics).
\end{abstract}

Mathematics subject classification (2000): 60E15, 60G50, 60G42, 60G48, 26A48, $26 \mathrm{D} 10$.

Key words and phrases: Upper bounds, probability inequalities, bounded random variables, Rademacher random variables, sums of independent random variables, (super)martingales, self-normalized sums, $t$ statistics.

\section{REFERENCES}

[1] V. BentKus, A remark on the inequalities of Bernstein, Prokhorov, Bennett, Hoeffding, and Talagrand, Lithuanian Math. J., 42, (2002), 262-269.

[2] V. BENTKUS, An inequality for tail probabilities of martingales with differences bounded from one side, J. Theoret. Probab., 16, (2003), 161-173.

[3] V. BentKus, On Hoeffding's inequalities, Ann. Probab., 32, (2004), 1650-1673.

[4] S. G. BoBKOv, F. GÖTZE, C. HoudRÉ, On Gaussian and Bernoulli covariance representations, Bernoulli, 7, (2002), 439-451.

[5] M. L. EATON, A probability inequality for linear combinations of bounded random variables, Ann. Statist., 2, (1974), 609-614.

[6] M. L. EAton, B. Efron, Hotelling's $T^{2}$ test under symmetry conditions, J. Amer. Statist. Assoc., 65 , (1970), 702-711.

[7] D. EdELMAN, An inequality of optimal order for the tail probabilities of the $T$ statistic under symmetry, J. Amer. Statist. Assoc., 85, (1990), 120-122.

[8] B. EFRON, Student's t test under symmetry conditions, J. Amer. Statist. Assoc., 64, (1969), $1278-1302$.

[9] S. E. GRAVERSEN, G. PEŠKIR, Extremal problems in the maximal inequalities of Khintchine, Math. Proc. Cambridge Philos. Soc., 123, (1998), 169-177.

[10] U. HaAgERUP, The best constants in the Khinchine inequality, Studia Math., 70, (1982), 231-283.

[11] W. HoEFFDING, Probability inequalities for sums of bounded random variables, J. Amer. Statist. Assoc., 58, (1963), 13-30.

[12] G. A. Hunt, An inequality in probability theory, Proc. Amer. Math. Soc., 6, (1955), 506-510.

[13] A. KHINCHIN, Über dyadische Brüche, Math. Z., 18, (1923), 109-116.

[14] G. PešKIR, A. N. ShIRYAEV, The Inequalities of Khintchine and Expanding Sphere of Their Action, Russian Math. Surveys, 50, (1995), 849-904.

[15] I. PINELIS, Extremal probabilistic problems and Hotelling's $T^{2}$ test under symmetry condition, (1991), Preprint, URL: http: / /arxiv.org/abs/math/0701806.

[16] I. PINELIS, Extremal probabilistic problems and Hotelling's $T^{2}$ test under a symmetry condition, Ann. Statist., 22, (1994), 357-368.

[17] I. PINELIS, Optimal tail comparison based on comparison of moments, in High dimensional probability (Oberwolfach, 1996), Progr. Probab., Birkhäuser, Basel, 43, (1998), 297-314. 
[18] I. PINELIS, Fractional sums and integrals of $r$-concave tails and applications to comparison probability inequalities, in Advances in stochastic inequalities (Atlanta, GA, 1997), Contemp. Math., Amer. Math. Soc., Providence, RI, 234, (1999), 149-168.

[19] I. PINELIS, On exact maximal Khinchine inequalities, in High dimensional probability, II (Seattle, WA, 1999), Progr. Probab., Birkhäuser Boston, Boston, MA, 47, (2000), 49-63.

[20] I. PINELIS, L'Hospital type rules for oscillation, with applications, J. Inequal. Pure Appl. Math., 2 , (2001), no. 3, Article 33, 24 pp. (electronic).

[21] I. PINELIS, Monotonicity properties of the relative error of a Padé approximation for Mills' ratio, J. Inequal. Pure Appl. Math., 3, (2002), no. 2, Art. 20, 8 pp. (electronic).

[22] I. PINELIS, L'Hospital type rules for monotonicity: applications to probability inequalities for sums of bounded random variables, J. Inequal. Pure Appl. Math., 3, (2002), no. 1, Article 7, 9 pp. (electronic).

[23] I. PINELIS, Dimensionality reduction in extremal problems for moments of linear combinations of vectors with random coefficients, in Stochastic inequalities and applications, Progr. Probab., Birkhäuser, Basel, 56, (2003), 169-185.

[24] I. PINELIS, Binomial upper bounds on generalized moments and tail probabilities of (super)martingales with differences bounded from above, in IMS Lecture Notes-Monograph Series, High Dimensional Probability, Institute of Mathematical Statistics, 51, (2006). DOI: 10.1214/074921706000000743. URL: http: //arxiv.org/abs/math.PR/0512301.

[25] I. PINELIS, On normal domination of (super)martingales, Electronic J. Probab., 11, (2006), Paper 39, 1049-1070. URL: http://www.math.washington.edu/ ejpecp/viewarticle.php?id=1648\& layout=abstract.

[26] I. PINELIS, On l'Hospital-type rules for monotonicity, J. Inequal. Pure Appl. Math., 7, (2006), no. 2, Article 40 (electronic).

[27] I. PINELIS, (2006). Exact inequalities for sums of asymmetric random variables, with applications, Probab. Theory Related Fields 139, (2007), no. 3-4, 605-635.

[28] I. PINELIS, (2007). Toward the best constant factor for the Rademacher-Gaussian tail comparison, ESAIM Probab. Stat., 11, (2007), 412-426 (electronic).

[29] P. WhitTLE, Bounds for the moments of linear and quadratic forms in independent variables, Teor. Verojatnost. i Primenen., 5, (1960), 331-335. 